\title{
Stochastic growth of radial clusters: weak convergence to the asymptotic profile and implications for morphogenesis
}

\author{
Carlos Escudero \\ Departamento de Economía Cuantitativa $\&$ \\ Instituto de Ciencias Matemáticas (CSIC-UAM-UC3M-UCM), \\ Universidad Autónoma de Madrid, \\ Ciudad Universitaria de Cantoblanco, \\ 28049 Madrid, Spain
}

\begin{abstract}
The asymptotic shape of randomly growing radial clusters is studied. We pose the problem in terms of the dynamics of stochastic partial differential equations. We concentrate on the properties of the realizations of the stochastic growth process and in particular on the interface fluctuations. Our goal is unveiling under which conditions the developing radial cluster asymptotically weakly converges to the concentrically propagating spherically symmetric profile or either to a symmetry breaking shape. We demonstrate that the long range correlations of the surface fluctuations obey a self-affine scaling and that scale invariance is achieved by means of the introduction of three critical exponents. These are able to characterize the large scale dynamics and to describe those regimes dominated by system size evolution. The connection of these results with mathematical morphogenetic problems is also outlined.

PACS numbers: 05.40.-a, 05.65.+b, 87.10.-e, 87.10.Mn
\end{abstract}




\section{INTRODUCTION}

The building blocks of mathematical morphogenesis were put several decades ago in the seminal works of Thompson [1] and Turing [2]. A particularly relevant problem in this context is the examination of the properties relating to the architecture of cell colonies, as already noted and investigated by Eden [3, 4]. At some basic underlying level, one could say that Turing and Eden shared the goal of understanding how a macroscopic structure, in particular one breaking the initial homogeneity, could arise out of a multiplicity of simple interactions. In the introduction of [2] one can read Turing thoughts on how a structure composed by randomly and isotropically proliferating cells could or could not break the initial spherical symmetry. But then his approach moved to the use of reaction-diffusion equations and to the search of a deterministic mechanism, the currently well known Turing instability, able to give rise to pattern formation. On the other hand, Eden focused his interest on the evolution of a pure growth process. He concentrated on a probabilistic abstraction of a developing cell colony and studied stochastic symmetry breaking concomitant to growth. In particular, Eden studied the architecture of a lattice cell colony to which new cells were added following certain probabilistic rules. The objective was determining the asymptotic colony profile. If the growth rules are isotropic then the realizations of the growth process are either spherically symmetric or this spherical symmetry is broken by means of long range fluctuations.

These sorts of discrete models are usually studied using continuum equations for ease of analytical treatment [5, 6]. While most of these developments have been carried out for systems in which there is an external input of mass, the universality of the surface fluctuations is expected to remain unchanged when the origin of mass appearance is internal. In fact, the Eden model in a strip geometry has been satisfactorily analyzed within the classical theoretical framework [7]. And, as we already have mentioned, this model was introduced as a probabilistic abstraction of a proliferating cell colony. So we expect our results to hold independently of the internal or external origin of mass input into the system. This will be so at least in those cases in which the growing radial interface can be described as a Monge patch in spherical coordinates. Growing clusters as the ones generated by diffusionlimited aggregation processes yield more complex structures that cannot be described in such simple terms, and thus fall beyond the scope of the present approach. 
Indeed, the original Eden problem can be greatly generalized by means of the use of stochastic partial differential equations. They allow a systematic study of the properties of the colony periphery, particularly of the interface fluctuations. At the same time, they allow us to get rid of the undesirable lattice anisotropy. In this work we will concentrate on the properties of the realizations of the stochastic growth process. Our goal is unveiling under which conditions the developing radial cluster asymptotically weakly converges to the concentrically propagating spherically symmetric profile. Let us emphasize that we are interested in determining the presence or absence of spherical symmetry in every realization of the stochastic growth process in the long time limit. As we are considering isotropic growth which is free from deterministic instabilities, averaging the cluster profile over many realizations yields an immediate spherical symmetry. The properties of the realizations can be ascertained, as we will see, calculating suitable correlations adapted from classical elements of stochastic growth theory [7, and keeping in mind that we should expect weak rather than strong convergence of the asymptotic profiles. Weak convergence is a mathematically totally precise concept which physically corresponds to the convergence resulting from the self-averaging of wildly oscillating quantities or structures [8].

Apart from the overall shape, the microscopic fluctuations of growing radial clusters have been studied as well. It turns out that these fluctuations sculpture a fractal surface which is statistically self-affine [7, 9, 10]. This microscopic roughness, in those cases in which the pointwise width [11] is well defined (which actually rules out all flat interfaces due to the microscopic properties of white noise) is described by the familiar scaling of planar systems [12]. This is not so, however, for long range fluctuations [13]. Long range radial correlations cannot be in general deduced from planar scalings. They still adopt a self-affine form for macroscopic length scales, but scale invariance is this time achieved for different values of the critical exponents. The following sections will discuss the numerical values and physical meaning of these exponents. We will see that they are able to characterize which

regimes asymptotically weakly converge to the radially symmetric shape and which do not.

\section{RADIAL CORRELATIONS}

In planar situations of non-equilibrium growth the interface is parameterized by the height function $h(x, t)$, which indicates the displacement of the interface with respect to some 
hyperplane taken as origin, at some $d$-dimensional spatial point $x$ and for some instant of time $t$ [7]. We illustrate this geometry in Fig. (1), where we present the one-dimensional solution to the so-called random deposition equation

$$
\partial_{t} h=F+\xi(x, t)
$$

where $\xi$ is a zero-mean Gaussian noise whose correlation is $\left\langle\xi(x, t) \xi\left(x^{\prime}, t\right)\right\rangle=\epsilon \delta\left(x-x^{\prime}\right) \delta\left(t-t^{\prime}\right)$. This numerical approximation is found by means of spatially discretizing the system.

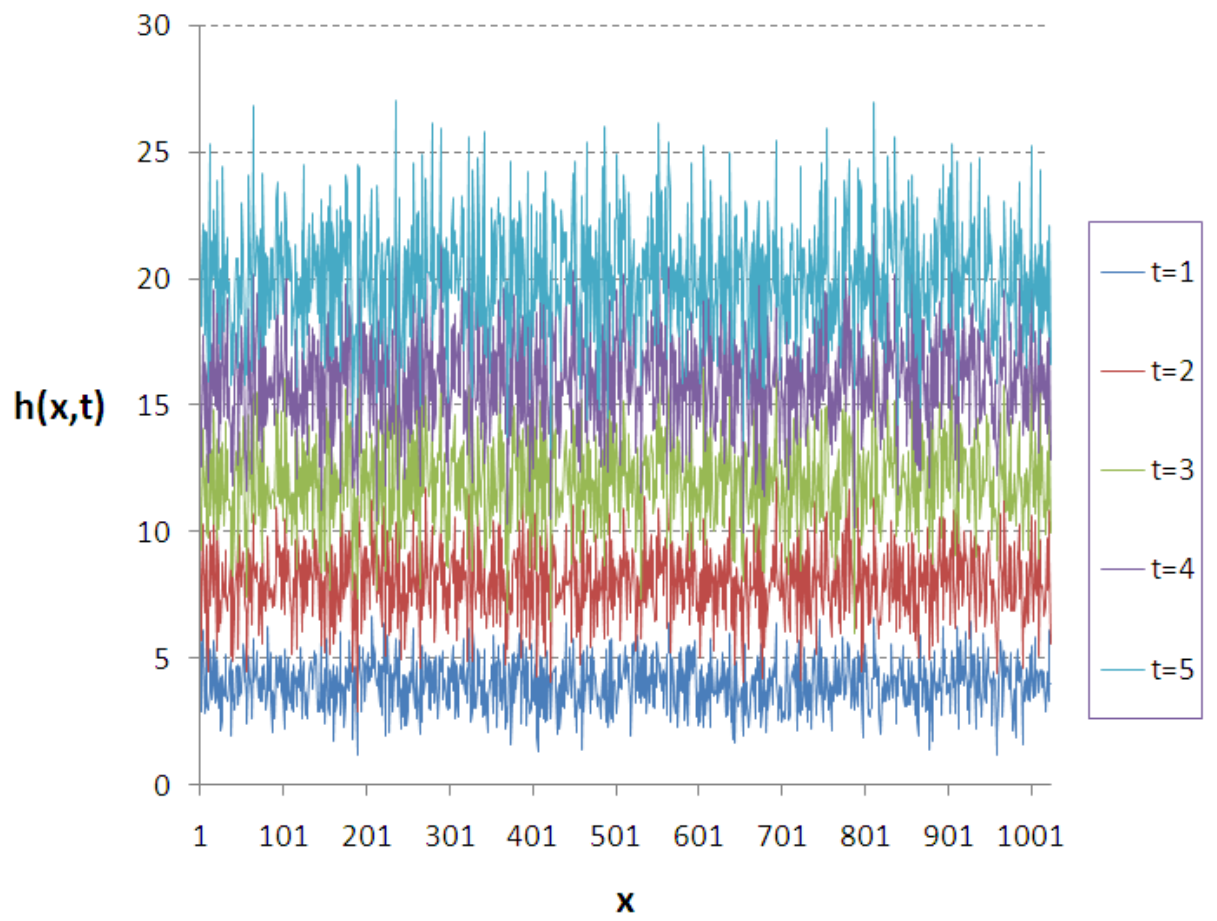

FIG. 1: Solution to the random deposition equation (1) at several times. The values of the parameters are $F=4, \epsilon=1$, and the system has been discretized in $2^{10}$ spatial points. We have used as initial condition $h(x, 0)=0$.

In these systems and for large spatiotemporal scales the height-height correlation function adopts the self-affine scaling form

$$
\begin{aligned}
\left\langle h(x, t) h\left(x^{\prime}, t\right)\right\rangle & =t^{2 \beta_{c}} f\left(\frac{\left|x-x^{\prime}\right|}{t^{1 / z_{c}}}\right)= \\
& =\left|x-x^{\prime}\right|^{2 \alpha_{c}} g\left(\frac{\left|x-x^{\prime}\right|}{t^{1 / z_{c}}}\right),
\end{aligned}
$$

which is invariant to the transformation $x \rightarrow b x, t \rightarrow b^{z} t$ and $h \rightarrow b^{\alpha} h$, for $\alpha=\beta z$ and $\alpha=\alpha_{c}, \beta=\beta_{c}$ and $z=z_{c}$, where the subscript $c$ denotes the classical value of an exponent 
for a given model. $\alpha$ is known as roughness exponent, $\beta$ as growth exponent and $z$ as dynamic exponent, $f$ and $g$ are scaling functions. This scaling is valid for a large class of linear and nonlinear models. If we consider the linear Langevin equation

$$
\partial_{t} h=-D|\nabla|^{\zeta} h+\xi(x, t)
$$

where $\xi$, as before, is a zero-mean Gaussian noise whose correlation is $\left\langle\xi(x, t) \xi\left(x^{\prime}, t\right)\right\rangle=$ $\epsilon \delta\left(x-x^{\prime}\right) \delta\left(t-t^{\prime}\right)$ and $|\nabla|^{\zeta}$ is a pseudo-differential operator to be understood in the Fourier transform sense, the exponents read $z=\zeta, \alpha=(\zeta-d) / 2$ and $\beta=1 / 2-d /(2 \zeta)$. One would like to know if the radial counterpart of Eq. (3) displays an analogous behavior to that dictated by scaling (2). In the radial extension of the theory one has a new degree of freedom which was trivial in the planar case: the rate of growth. If we assume that the average radius (measured as distance from the origin) of the macroscopic radial form grows as a power law $\langle r\rangle=F t^{\gamma}$ for $t \geq t_{0}$ and a growth index $\gamma>0$, then the equation for the radial surface fluctuation $\rho(\theta, t)$ is

$$
\partial_{t} \rho=-\frac{D}{F^{\zeta} t^{\zeta \gamma}}\left|\nabla_{\theta}\right|^{\zeta} \rho+\frac{1}{F^{d / 2} t^{\gamma d / 2}} \frac{\eta(\theta, t)}{J(\theta)^{1 / 2}},
$$

according to the reparametrization invariance principle [14, 15], and where $\theta$ denotes the set of angles parameterizing the $d$-dimensional radial interface and $J(\theta)$ is the Jacobian determinant of the change of variables from $(x, h)$ to $(\theta, r)$ evaluated at $r=1$ [16]. The noise is simply $\eta=\xi / \sqrt{\epsilon}$. In order to visually compare planar and radial processes we have plotted the field $r(\theta, t)=\langle r\rangle+\sqrt{\epsilon} \rho(\theta, t)$ for $D=0$ in Fig. (2). Neglecting the diffusion constant makes this stochastic process the radial counterpart of the random deposition process plotted in Fig. (1). We will therefore refer to it as the radial random deposition process.

As we have mentioned in the introduction, one of the motivations of this work is studying the large scale, long time properties of a randomly proliferating set of cells or entities in general. Such a process can be modelled in some detail using a master equation, which can be mapped into a stochastic partial differential equation provided with a multiplicative noise term [17, 18]. On the other hand, we focus in this work on stochastic partial differential equations provided with additive noise. While this could seem as an apparent paradox, the underlying reason is that equations (3) and (4) are effective models. So they are intended to describe just the long range properties of the growing interface, and in this limit fluctuations 


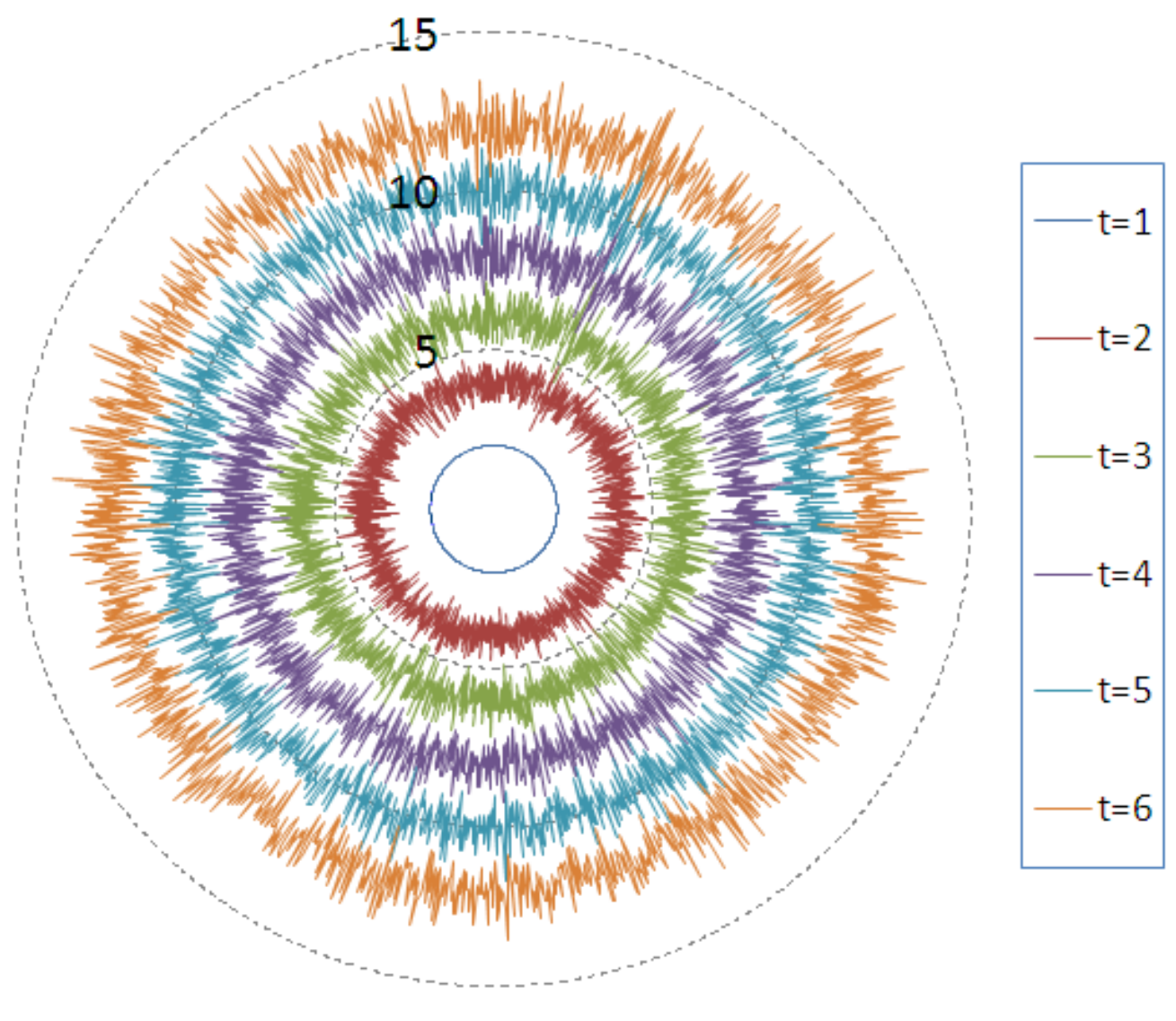

FIG. 2: Radial random deposition growth process plotted at different times. The values of the parameters are $F=2, \gamma=1, \epsilon=(200 \pi)^{-1}$, and the system has been discretized in $2^{11}$ spatial points. We have used as initial condition $r(\theta, 1)=2$ (note that we have used as initial time $t_{0}=1$ ).

can be effectively considered additive. A nice example of this, a case in which a front driven by multiplicative noise is found to belong to a universality class described by an additive noise stochastic equation, is described in [19].

The analysis of the exact solutions to Eq. (4) for $\gamma>1 / \zeta$ and long times shows the diffusion term is irrelevant in the large scale $\left|\theta-\theta^{\prime}\right| \gg t^{1 / \zeta-\gamma}$ and the correlation becomes [15, 16 ]

$$
C\left(\theta, \theta^{\prime}, t\right) \sim\left\{\begin{array}{lll}
t^{1-\gamma d} \delta\left(\theta-\theta^{\prime}\right) / J(\theta) & \text { if } & \gamma d<1, \\
\ln (t) \delta\left(\theta-\theta^{\prime}\right) / J(\theta) & \text { if } & \gamma d=1, \\
\delta\left(\theta-\theta^{\prime}\right) / J(\theta) & \text { if } & \gamma d>1,
\end{array}\right.
$$

where $C\left(\theta, \theta^{\prime}, t\right)=\left\langle\rho(\theta, t) \rho\left(\theta^{\prime}, t\right)\right\rangle$. In terms of arc-length differences $\ell-\ell^{\prime} \sim t^{\gamma}\left(\theta-\theta^{\prime}\right)$ one 
finds [16]

$$
C\left(\ell, \ell^{\prime}, t\right) \sim\left\{\begin{array}{llc}
t \delta\left(\ell-\ell^{\prime}\right) / J\left(t^{-\gamma} \ell\right) & \text { if } & \gamma d<1, \\
t \ln (t) \delta\left(\ell-\ell^{\prime}\right) / J\left(t^{-\gamma} \ell\right) & \text { if } & \gamma d=1, \\
t^{\gamma d} \delta\left(\ell-\ell^{\prime}\right) / J\left(t^{-\gamma} \ell\right) & \text { if } & \gamma d>1 .
\end{array}\right.
$$

This correlation is invariant to the transformation $\ell \rightarrow b \ell, t \rightarrow b^{z} t$ and $\rho \rightarrow b^{\alpha} \rho$ for $z=$ $1 / \gamma=: z_{r}$ and $\alpha=1 /(2 \gamma)-d / 2=: \alpha_{r}\left(\alpha=0=: \alpha_{r}\right)$ when $\gamma d<1(\gamma d \geq 1)$. We define

$\beta_{r}:=\alpha / z=\alpha_{r} / z_{r}=(1-\gamma d) / 2\left(\beta_{r}=0\right)$ when $\gamma d<1(\gamma d \geq 1)$. First, the functional form of this correlation is not reducible to scaling form (2), and second, the exponents are totally different (this makes precise the analysis carried out in [13]). In summary, long range radial surface fluctuations are not describable in terms of scaling (2).

\section{DILUTION AND UNIVERSALITY CLASS BIFURCATION}

It is clarifying to consider a simplified problem: an abstraction in which Eq. (3) is placed on a growing domain [20]. If this is realized by means of the transformation $x \rightarrow\left(t / t_{0}\right)^{\gamma} x$ one finds

$$
\partial_{t} h=-D\left(\frac{t_{0}}{t}\right)^{\zeta \gamma}|\nabla|^{\zeta} h+\gamma F t^{\gamma-1}+\left(\frac{t_{0}}{t}\right)^{d \gamma / 2} \xi
$$

where the mass source is explicitly included and we assume fast growth $\gamma>1 / \zeta$ and the rough interface inequality $\zeta>d$. In this case it can be shown that the correlation obeys scaling (2) for short length scales $\left|x-x^{\prime}\right| \ll t^{(1-\zeta \gamma) / \zeta}$ and for long length scales $\left|x-x^{\prime}\right| \gg$ $t^{(1-\zeta \gamma) / \zeta}$ it is $[20]$

$$
C\left(y, y^{\prime}, t\right) \sim\left\{\begin{array}{llc}
t \delta\left(y-y^{\prime}\right) & \text { if } & \gamma d<1 \\
t \ln (t) \delta\left(y-y^{\prime}\right) & \text { if } & \gamma d=1 \\
t^{\gamma d} \delta\left(y-y^{\prime}\right) & \text { if } & \gamma d>1
\end{array}\right.
$$

where $y=\left(t / t_{0}\right)^{\gamma} x$ is the counterpart of the arc-length coordinate. This correlation is scale invariant for the exponents $\left\{\alpha_{r}, z_{r}\right\}$ but not for the exponents $\left\{\alpha_{c}, z_{c}\right\}$.

This way of realizing domain growth agrees with reparametrization invariance [16], but is not unique. It makes space and mass grow simultaneously [16], while it is possible to realize domain growth at constant mass by introducing a dilution term [20, 21]

$$
\partial_{t} h=-D\left(\frac{t_{0}}{t}\right)^{\zeta \gamma}|\nabla|^{\zeta} h-\frac{d \gamma}{t} h+\gamma F t^{\gamma-1}+\left(\frac{t_{0}}{t}\right)^{d \gamma / 2} \xi
$$


In this case the correlation is

$$
C\left(y, y^{\prime}, t\right) \sim t \delta\left(y-y^{\prime}\right)
$$

which is scale invariant for both $\left\{\alpha_{c}, z_{c}\right\}$ and $\left\{\tilde{\alpha}_{r}=1 /(2 \gamma)-d / 2, z_{r}\right\}$. The first invariance can be used to argue that this correlation is a direct consequence of scaling (2), but the scaling form (10) is definitely ambiguous in view of the second invariance. Both sort of dynamics, with and without dilution, are physically realizable; while dilution dynamics is apparently better suited to describe some natural processes, no-dilution dynamics seems to agree better with the numerical simulation of some discrete models as discussed in [16]. Together with the difference in the roughness exponent, very pronounced differences in the auto-correlation and persistence exponents separate the interface behavior in presence and absence of dilution [20]. This reveals that the non-uniqueness of the extension of a Langevin equation to a growing domain implies in turn a universality class bifurcation.

\section{GEOMETRIC CONSTRAINTS}

One can apply what has been learned in the previous section about growing domains to the radial situation. Including dilution in the radial equation for interface motion yields a different dynamics from the one established by reparametrization invariance. The correlation in this case becomes [16]

$$
C\left(\ell, \ell^{\prime}, t\right) \sim t \frac{\delta\left(\ell-\ell^{\prime}\right)}{J\left(t^{-\gamma} \ell\right)}
$$

which is scale invariant for the exponents $\left\{\tilde{\alpha}_{r}, z_{r}\right\}$ but not for $\left\{\alpha_{c}, z_{c}\right\}$. The difference among $\alpha_{r}$ and $\tilde{\alpha}_{r}$ originates in the memory effects that affect the surface described by reparametrization invariance and disappear by virtue of dilution [16, 20, 22]. The difference between the sets $\left\{\alpha_{r}, z_{r}\right\}$ and $\left\{\tilde{\alpha}_{r}, z_{r}\right\}$, and the classical set $\left\{\alpha_{c}, z_{c}\right\}$ is of a different nature. Note that the Langevin dynamics on a growing domain and influenced by dilution gave rise to the random deposition correlation 100 :

$$
\left\langle h(y, t) h\left(y^{\prime}, t\right)\right\rangle \sim t \delta\left(y-y^{\prime}\right)
$$

This correlation is invariant to the transformation

$$
y \rightarrow b y, \quad t \rightarrow b^{z} t, \quad \text { and } \quad h \rightarrow b^{\alpha} h
$$


for

$$
z=\kappa \quad \text { and } \quad \alpha=(\kappa-d) / 2, \quad \kappa \in \mathbb{R} ;
$$

let us remark that scale invariance is achieved for any real number $\kappa$, thus (13) and (14) actually constitute a one-parameter family of scale transformations. This is, there is one degree of freedom in the critical exponents, or in other words, the scaling form is insensitive to the dynamic exponent. Contrarily, in a radial geometry the correlation, Eq. (11), is scale invariant only if $\kappa=1 / \gamma$ : this is the constraint imposed by the geometrical factor $J$. The Jacobian determinant $J=\sin \left(\theta_{1}\right)^{d-1} \sin \left(\theta_{2}\right)^{d-2} \cdots \sin \left(\theta_{d-1}\right)$ for a $d$-dimensional interface, where $\theta_{1}, \cdots, \theta_{d-1}$ is the set of polar angles, while it is of course independent of the azimuth angle $\theta_{d}$. So this constraint appears for $d \geq 2$, but it is not present for $d=1$. The reason is that the one-dimensional radial interface is topologically identical to the one-dimensional, topologically toroidal, interface which results from assuming periodic boundary conditions on the growing domain setting. In this case, the angle-like coordinate $x$, to which the azimuth angle is akin, may be either dimensional or dimensionless (this could be expressed by means of the dichotomy of Lagrangian/Eulerian coordinates similarly to the developments in [20]). On the other hand, the polar angles, ranging only $\pi$ radians, cannot be associated to periodic coordinates which could have dimensions. This fixes the scaling $z=1 / \gamma$.

One can use the ambiguity of Eq. (12) to claim that $z=\zeta$ is a good dynamic exponent in this case, but that would be accepting that topology can modify the scaling. Also, the value of the auto-correlation exponent in this case suggests that $z=1 / \gamma$ is the good dynamic exponent [20]. In any case, it is now clear that there is no basis for claiming that correlation (10)- 12 is a consequence of scaling (2) in the present framework. This is because, although both forms are invariant to the transformation (13) for $z=\zeta$ (for linear equations like (3)), self-similar form (10)-12 preserves scale invariance independently of the value of $z$ (as specified in Eq. (14)). So this second form is much more general and the value $z=\zeta$ plays no special role in it (let us note again that the value $z=1 / \gamma$ plays a special role in the sense that it appears in the auto-correlation function [20]). Despite this ambiguity in the growing domain situation, it is clear that the radial correlation (11) is just invariant for $\left\{\tilde{\alpha}_{r}, z_{r}\right\}$, and the classical exponents $\left\{\alpha_{c}, z_{c}\right\}$ play no role in this case. This can be seen by means of a direct application of transformation (13) to this correlation and substituting $\alpha$ and $z$ for the two different alternatives. 


\section{DIMENSIONAL ANALYSIS AND NONLINEAR DYNAMICS}

Our results so far allow us to develop a consistent dimensional analysis of radial equations. We start with Eq. (4) performing the scale transformation $\rho \rightarrow b^{\alpha} \rho$ and $t \rightarrow b^{z} t$. The angular variables are dimensionless and this, as specified by the factor $J$, fixes the exponent $z=1 / \gamma$. The stochastic term becomes scale invariant for $\alpha=1 /(2 \gamma)-d / 2$ and the diffusion irrelevant for $\gamma>1 / \zeta$ : this agrees with our previous analysis using the explicit solutions of the linear equations. We can now use this technique to get some insight on nonlinear equations. The Kardar-Parisi-Zhang (KPZ) equation [23] for the radial surface fluctuation can be written as

$$
\partial_{t} \rho=\frac{\nu}{F^{2} t^{2 \gamma}} \partial_{\theta}^{2} \rho+\frac{\lambda}{F^{2} t^{2 \gamma}}\left(\partial_{\theta} \rho\right)^{2}+\frac{1}{F^{d / 2} t^{\gamma d / 2}} \frac{\eta(\theta, t)}{J(\theta)^{1 / 2}} .
$$

We note that scaling arguments were successfully employed in determining the critical behavior of KPZ with a time dependent coefficient of the nonlinearity [24]. If we select the same values of $\alpha$ and $z$ as in the linear case in order to make the stochastic term scale invariant we find that diffusion and nonlinearity are irrelevant for $\gamma>1 / 2$ and $\gamma>3 /(4+d)$ respectively. This agrees with the naif dimensional analysis of KPZ and thus we cannot expect it to be correct. In fact, the naïf dynamical exponent $z=(4+d) / 3$ found by means of a dimensional analysis of the KPZ nonlinearity does not agree with the one measured in simulations and successfully predicted by the dynamic renormalization group analysis in one dimension [7]. It is plausible that the nonlinearity interacts with the noise to yield a correlation different from (6) (or from (11) if dilution is included). In order to guess what form the correlation would have we can try to get some illumination from scaling (2). Taking the short time limit in (2) we find

$$
C\left(x, x^{\prime}, t\right) \sim t^{2 \beta_{c}+d / z_{c}} \delta\left(x-x^{\prime}\right)
$$

where the temporal prefactor expresses the time dependence of the variance of the interface center of mass position [16]. Using this form we modify, for instance, Eq. (11) to the new correlation

$$
C\left(\ell, \ell^{\prime}, t\right) \sim t^{2 \beta_{c}+d / z_{c}} \frac{\delta\left(\ell-\ell^{\prime}\right)}{J\left(t^{-\gamma} \ell\right)},
$$

which yields the exponents $\alpha=\left(2 \beta_{c}+d / z_{c}\right) /(2 \gamma)-d / 2, z=1 / \gamma$ and $\beta_{r}=\left(2 \beta_{c}+d / z_{c}\right) / 2-$ $d \gamma / 2$. In this case we have the diffusion is irrelevant whenever $\gamma>1 / 2$ and the nonlinearity is irrelevant if $\gamma>1 / z_{c}$, where we have employed the relation $\alpha_{c}+z_{c}=2$ in the derivation of 
the last inequality. This last inequality could be considered as desirable but correlation (17) has a different drawback. As we have said, the exponent $\beta_{r}$ describes the time power law dependence of the standard deviation of the interface center of mass position. For the Eden model, which in planar format belongs to the KPZ universality class [7], $d=\gamma=1$ and so $\beta_{r}=1 / 6$. This exponent however does not agree with the one measured in simulations [25] and this constitutes another disadvantage of the use of scaling (2) in radial systems. We note that considering the planar KPZ equation on a growing domain does not simplify much things with respect to considering the full radial case [26].

Together with the KPZ equation, the Villain-Lai-Das Sarma (VLDS) equation [27, 28] is one of most important nonlinear models for surface growth. If one derives the VLDS equation in the radial setting one finds irrelevance of the diffusion for $\gamma>1 / 4$ and of the nonlinearity for $\gamma>1 / z_{c}$. In this case the well known hyperscaling relation for conserved growth models yields $2 \beta_{c}+d / z_{c}=1$, and so equality of (17) with (11). This suggests that the scaling properties derived herein for linear models are also valid for some nonlinear models as the VLDS equation.

\section{CONCLUSIONS}

In this work we have examined the dynamic scaling of radial interfaces. We have shown that long range fluctuations, which are describable by two-point correlation functions, become scale invariant for values of the critical exponents which may totally differ from the corresponding ones in a planar geometry. In rapidly growing radial systems we have found

$$
\alpha=\frac{1}{2 \gamma}-\frac{d}{2}, \quad z=\frac{1}{\gamma}, \quad \beta_{r}=\frac{1}{2}-\frac{\gamma d}{2},
$$

all of which have a clear physical meaning. The $\beta_{r}$ exponent, as we have discussed, describes the interface center of mass fluctuations. The $\alpha$ exponent associated to the radial surface fluctuation indicates how much the growing cluster deviates from a $d$-dimensional growing hypersphere. For $\alpha<0$ the growing cluster converges to a hypersphere concentrically growing at the deterministic rate $t^{\gamma}$, and in this sense the growth profile is flat. This sort of convergence is of course weak: the profile converges to the hypersphere on (any type of spatial) average. The microscopic roughness, which is given by the height-difference correlation, reduces to that of the planar profile [20] and precludes strong or pointwise 
convergence. This is so at least in situations in which the height-difference correlation is well defined; otherwise a different scaling is possible even locally in space [16]. This makes precise and generalizes our previous results [13] in the following way: a fast growth rate will make the interface weakly converge to a hypersphere. We have further found the scaling relation $\alpha=\beta_{r} z$, which quantitatively relates the center of mass fluctuations, growth rate and rate of (weak) convergence to/divergence from the hyperspherical profile. These results, together with our previous ones [16, 20], suggest the following generalization of the critical exponents for arbitrary values of $\gamma$

$$
\alpha=\frac{z-d}{2}, \quad \beta:=\frac{\alpha}{z}=\frac{1}{2}-\frac{d}{2 z}, \quad \text { for } \quad z=\min \left\{z_{c}, \frac{1}{\gamma}\right\},
$$

which should be valid for linear models as well as some nonlinear equations as the VLDS one, which present a conserved drift and its critical behavior is accessible to dimensional analysis. For the KPZ equation, which nonlinearity introduces both mass and fluctuations into the system, we expect a different behavior. As we have seen, the amount of mass entering the interface (and of course the way it is introduced) may change the critical behavior. We have explicitly shown this by means of the linear models in absence and presence of dilution, which gave respectively rise to correlations (6) and (11).

\begin{tabular}{|c|c|c|c|}
\hline$\gamma<\frac{1}{z_{c}}$ & $\frac{1}{z_{c}}<\gamma<\frac{1}{d}$ & $\frac{1}{z_{c}}<\gamma<\frac{1}{d+2}$ & $\gamma>\max \left\{\frac{1}{d}, \frac{1}{z_{c}}\right\}$ \\
\hline Classical exponents & Roughness & Super-roughness & Weak convergence to the hyperspherical profile \\
\hline
\end{tabular}

TABLE I: Morphologies for different spatial dimensions and exponents.

We have summarized the different morphologies that can appear in Table II (see also Fig. 2 in [16]). In the first column, for small $\gamma$, we have placed the regime in which the classical values of the exponents are recovered. The other three columns represent situations in which this is not the case. The "roughness" column describes the situation in which the radial fluctuations do not average each other out for long times, so in this regime the overall appearance of the cluster deviates from the hyperspherical profile. The "super-roughness" column states a particular case of this last regime, in which the amplitude of the radial fluctuations grows faster than the arc-length sustaining them. In both cases roughness should be understood as the degree of weak divergence (as opposed to weak convergence) from the hyperspherical profile. Finally, the last column describes the regime in which the weak convergence to the hyperspherical profile is reached asymptotically in time. 
Note that the meaning of the exponent $\beta$ in (19) changes for $\gamma<1 / z_{c}$ and for $\gamma>$ $1 / z_{c}$. For $\gamma<1 / z_{c}$ this exponent describes the short time dependence of the spatially averaged pointwise width with time, as in the classical situation. For $\gamma>1 / z_{c}$, as we have mentioned, it describes the center of mass fluctuations, i. e., the less dominant source of fluctuations is chosen in each regime. For $\gamma<1 / z_{c}$ and $\alpha>0$ (which implies the height difference correlation is well defined and the pointwise width is finite) there are always center of mass fluctuations. For $\gamma>1 / z_{c}$ the situation is different as it is possible to find growth regimes with no center of mass fluctuations and which pointwise width increases as a power law of time. This is precisely the weak converge to the hyperspherical profile: while microscopically the interface is rough, macroscopically it is a concentrically propagating hypersphere. The change of meaning of the exponent $\beta$ coincides with a change of physics. The long range shape of the interface is no longer affected by the diffusion mechanism, which becomes irrelevant, but for the system size evolution, which sculptures the resulting profile [20]. And so, the violation of scaling (2) is associated with the advent of a new dominant physical mechanism in the interface macroscale: system size growth overtakes diffusion and determines the cluster macroscopic shape.

\section{Acknowledgments}

This work has been partially supported by the MICINN (Spain) through Project No. MTM2010-18128.

[1] D. W. Thompson, On Growth and Form (Cambridge University Press, Cambridge, 1917).

[2] A. M. Turing, Phil. Trans. R. Soc. B 237, 37 (1952).

[3] M. Eden, in Symposium on Information Theory in Biology, edited by H. P. Yockey (Pergamon Press, New York, 1958).

[4] M. Eden, in Proceedings of the Fourth Berkeley Symposium on Mathematical Statistics and Probability, edited by J. Neyman (University of California Press, Berkeley, 1961).

[5] C. A. Haselwandter and D. D. Vvedensky, Phys. Rev. Lett. 98, 046102 (2007).

[6] C. A. Haselwandter and D. D. Vvedensky, Phys. Rev. E 76, 041115 (2007). 
[7] A.-L. Barabási and H. E. Stanley, Fractal Concepts in Surface Growth (Cambridge University Press, Cambridge, 1995).

[8] Weak convergence (or convergence in the sense of distributions) is formalized in mathematical analysis texts as convergence in the dual space. Intuitively, it corresponds to the spatial self-averaging of a wildly oscillating quantity. A good example of this is given by the RiemannLebesgue lemma, which states the weak convergence of wildly oscillating trigonometric functions. Also, weak convergence is a relevant concept in physics: it might result, for instance, from the self-averaging of random fluctuations. As an example, some quantum mechanical quantities weakly converge to their classical counterparts.

[9] T. Halpin-Healy and Y.-C. Zhang, Phys. Rep. 254, 215 (1995).

[10] J. M. Hammersley and G. Mazzarino, Comb. Probab. Comput. 3, 471 (1994).

[11] The pointwise width is $W(t)=\left\{\int\left[h(x, t)-\int h\left(x^{\prime}, t\right) d x^{\prime}\right]^{2} d x\right\}^{1 / 2}$. The function height $h(x, t)$ is introduced in the following.

[12] F. Family and T. Vicsek, J. Phys. A 18, L75 (1985).

[13] C. Escudero, Phys. Rev. Lett. 100, 116101 (2008).

[14] M. Marsili, A. Maritan, F. Toigo, and J.R. Banavar, Rev. Mod. Phys. 68, 963 (1996).

[15] C. Escudero, Ann. Phys. 324, 1796 (2009).

[16] C. Escudero, Phys. Rev. E 84, 031131 (2011).

[17] E. Hernández-García and C. López, Phys. Rev. E 70, 016216 (2004).

[18] C. Escudero, J. Buceta, F. J. de la Rubia, and K. Lindenberg, Phys. Rev. E 69, 021908 (2004).

[19] E. Moro, Phys. Rev. Lett. 87, 238303 (2001).

[20] C. Escudero, J. Stat. Mech. P07020 (2009).

[21] E. J. Crampin, E. A. Gaffney, and P. K. Maini, Bull. Math. Biol. 61, 1093 (1999).

[22] C. Escudero, arXiv:0907.0898.

[23] M. Kardar, G. Parisi, and Y.-C. Zhang, Phys. Rev. Lett. 56, 889 (1986).

[24] E. Hernández-García, T. Ala-Nissila, and M. Grant, Europhys. Lett. 21, 401 (1993).

[25] S. C. Ferreira Jr. and S. G. Alves, J. Stat. Mech. (2006), P11007.

[26] H. S. Wio, C. Escudero, J. A. Revelli, R. R. Deza, and M. S. de la Lama, Phil. Trans. R. Soc. A 369, 396 (2011).

[27] J. Villain, J. Phys. I (France) 1, 19 (1991).

[28] Z.-W. Lai and S. Das Sarma, Phys. Rev. Lett. 66, 2348 (1991). 